\section{OP0174 PARTIAL KNEE REPLACEMENT IS ASSOCIATED WITH A LOWER RISK OF VENOUS THROMBOEMBOLISM AND OPIOID USE THAN TOTAL KNEE REPLACEMENT BUT INCREASED RISK OF LONG-TERM REVISION: A MULTINATIONAL, MULTI- DATABASE, PROPENSITY SCORE-MATCHED, COHORT ANALYSIS INCLUDING OVER 280,000 PATIENTS}

Daniel Prieto-Alhambra ${ }^{1}$, Edward Burn ${ }^{1}$, James Weaver ${ }^{2}$, Anthony G Sena ${ }^{2}$, Henry Morgan Stewart ${ }^{3}$, Patrick Ryan ${ }^{2} .{ }^{1}$ Centre for Statistics in Medicine, NDORMS, University of Oxford, Oxford, United Kingdom; ${ }^{2}$ Janssen Research and Development, Titusville, United States of America; ${ }^{3}$ IQVIA, London, United Kingdom

Background: Knee replacement is one of the few effective treatments for severe knee osteoarthritis. A number of surgical interventions are available, including two main types: partial or total knee replacement.

Objectives: We aimed to assess the outcomes of partial compared to total knee replacement.

Methods: We conducted a multi-database propensity score-matched cohort study. Data was obtained from 4 US claims databases (IBM MarketScan ${ }^{\circledR} \mathrm{Com}-$ mercial Database (CCAE), IBM MarketScan ${ }^{\circledR}$ Medicare Supplemental Database (MDCR), Optum ${ }^{\circledR}$ de-identified Clinformatics ${ }^{\circledR}$ Datamart, Extended - Date of Death (Optum), and Pharmetrics), and 1 UK primary care electronic medical record database (THIN).

All people aged 40 years or older at the time of knee replacement surgery were included. Data were mapped to a common data model (OMOP), and processed using analytical tools developed by the OHDSI community.

Participants were followed up from the date of their first knee replacement and for up to 5 years. Outcomes included short-term (60-day) post-operative complications (infection, venous thromboembolism, mortality, readmission), opioid use in the 3-12 months post-surgery as a proxy for persistent pain, and 5-year revision risk.

Propensity score matching (up to 1:10) was used to control for all available confounders, and negative control outcomes and calibration to minimize the impact of residual confounding. Separate Cox regression models were fitted for each outcome to estimate calibrated $\mathrm{HR}$ ( $\mathrm{cHR}$ ) and 95\% confidence intervals related to partial, using total knee replacement as a reference group.

Results: A total of 32,379 and 250,377 patients receiving partial and total knee replacement respectively were matched. Short-term risk of venous thromboembolism was consistently lower in the former, with cHR ranging from 0.47 [0.32-0.71] in MDCR to 0.76 [0.59-0.99] in CCAE. No differences were observed in the risk of other complications. The use of opioids in months 3 to 12 after surgery was also lower in subjects receiving partial knee replacement, with $\mathrm{cHR}$ ranging from 0.70 [0.57-0.90] in THIN to 0.86 [0.78-0.96] in Optum.

Conversely, risk of 5-year revision was consistently higher amongst those undergoing partial compared to total knee replacement: cHR ranged from 1.51 [1.241.88] in CCAE to 2.16 [1.63-3.15] in MDCR.

Conclusion: Partial knee replacement is associated with a $25 \%$ to $50 \%$ reduction in short-term risk of venous thromboembolism, and a 15\%-30\% lower risk of persistent pain post-surgery. In contrast, partial knee replacement results in a $50 \%$ to $>100 \%$ excess risk of revision in the longer term. This information should be used to individualize surgical indications for patients with severe knee osteoarthritis. Disclosure of Interests: : Daniel Prieto-Alhambra Grant/research support from: Grants from Amgen, UCB Biopharma and Servier outside the submitted work, Consultant for: UCB Biopharma, Speakers bureau: Amgen, Edward Burn: None declared, James Weaver Shareholder of: Shareholder in Johnson \& Johnson, Employee of: Full-time employee of Janssen Research \& Development, a pharmaceutical company of Johnson \& Johnson., Anthony G Sena Shareholder of: Shareholder in Johnson \& Johnson, Employee of: Full-time employee of Janssen Research \& Development, a pharmaceutical company of Johnson \& Johnson, Henry Morgan Stewart Employee of: Full-time employee of IQVIA, Patrick Ryan Shareholder of: Shareholder in Johnson \& Johnson, Employee of: Full-time employee of Janssen Research \& Development, a pharmaceutical company of Johnson \& Johnson.

DOI: 10.1136/annrheumdis-2019-eular.886

\section{OP0175 METHOTREXATE IN PATIENTS WITH HAND OSTEOARTHRITIS REFRACTORY TO ANALGESICS: A RANDOMISED, DOUBLE-BLIND, PLACEBO CONTROLLED TRIAL}

Stéphanie Ferrero ${ }^{1}$, Ruth Wittoek ${ }^{2}$, Edem Allado $^{3}$, Eric Fontas ${ }^{4}$, Coralie Cruzel $^{4}$, Liana Euller-Ziegler ${ }^{1}$, Damien Loeuille ${ }^{3}$, Christian Roux ${ }^{1} .{ }^{1}$ University of Nice cote d'azur/CHU Nice, Department of rheumatology, Nice, France; ${ }^{2}$ Ghent, Ghent, Belgium; ${ }^{3}$ Nancy, Nancy, France; ${ }^{4}$ Nice, Nice, France

Background: To date, no studies have been published on the effect of Methotrexate (MTX) in hand osteoarthritis (HOA).
Objectives: The aim of our study is to examine the effect of methotrexate on pain in symptomatic erosive $\mathrm{HOA}$, as well as its functional and structural effect using $\mathrm{MRI}$ and radiography.

Methods: This prospective, single-center, randomized, double-blind, placebocontrolled study followed patients with HOA over 12 months. Patients were randomized to Methotrexate $10 \mathrm{mg}$ per week or placebo.

The primary endpoint was pain assessment at 3 months, and secondary end points were clinical (Visual Analogue Scale "VAS" pain at 12 months), and radiographic (radiographic anatomical score, then the Ghent University Score System "GUSS" and MRI)

All subjects aged between 45 and 85 years, who had apparent NSAIDs and pain traitement failure with an VAS greater than 4/10, and at least one erosive joint were included.

Results: Out of 71 evalued patients with symptomatic erosive HOA, and 64 matched the inclusion criteria and were randomized. Among them, 32 patients were randomized in the placebo group and 32 in the Methotrexate group. There was no significant difference between the two groups on the primary endpoint at 3 months, with an average decrease in VAS in the placebo group of $8.36 \mathrm{~mm} \mathrm{(+/-}$ $25.15)$, and $17.47 \mathrm{~mm}(+/-28.37)$ in the treatment group $(p=0.1797)$. At 12 months, no difference was found in the evolution of pain between the two groups $(p=0.6045)$ as well as in the functional evolution. In subjects taking Methotrexate erosive joints move more to a remodeling phase undergoing treatment $(27 \%)$ than placebo subjects $(15 \%)(p=0.0278)$. They also seem to evolve less towards erosion when their joints present a space loss $(29 \%$ become erosive under placebo against $8 \%$ in the Methotrexate group) $(p=0.0879)$. No predictor of erosive outcome was found in patients with non-erosive hand osteoarthritis.

Conclusion: Our study does not show superior efficacy of MTX over placebo over pain and function in subjects with erosive HOA. Our results suggest a possible structural effect on the radiological evolution of HOA under Methotrexate.

Disclosure of Interests: None declared

DOI: 10.1136/annrheumdis-2019-eular.4373

\section{OP0176 HEALTH-RELATED QUALITY OF LIFE IN HAND OSTEOARTHRITIS PATIENTS FROM THE GENERAL POPULATION AND THE OUTPATIENT CLINIC}

Marieke Loef ${ }^{1}$, Wendy Damman ${ }^{1}$, Renée de Mutsert ${ }^{2}$, Frits Rosendaal ${ }^{2}$, Margreet Kloppenburg ${ }^{1,2}$. ${ }^{1}$ Leiden University Medical Center (LUMC), Rheumatology, Leiden, Netherlands; ${ }^{2}$ Leiden University Medical Center (LUMC), Epidemiology, Leiden, Netherlands

Background: In osteoarthritis (OA) patients health-related quality of life (HRQoL) is decreased. Whether patients with OA seeking care in a rheumatology outpatient clinic experience more impact on HRQoL than those in the general population is unknown.

Objectives: To investigate the impact of hand OA on physical and mental HRQoL in the general population, and to investigate the difference in impact between patients who have, and who have not been referred to a medical specialist.

Methods: In the population-based Netherlands Epidemiology of Obesity (NEO) study, middle-aged participants were recruited from the greater area of Leiden. In the Hand OSTeoArthritis in Secondary care (HOSTAS) study, patients with a rheumatologist's diagnosis of primary hand OA were recruited from the outpatient clinic at the Leiden University Medical Center, a secondary and tertiary referral center. In both cohorts, hand and knee OA was defined by the ACR clinical classification criteria. Patients with fibromyalgia or inflammatory rheumatic conditions were excluded. Fur the current analyses, only patients classified with hand OA alone were included. HRQoL was measured with the SF-36 questionnaire; we calculated standardized scores on a scale of 0 to 100 with subsequent application of a norm-based transformation (mean 50 , standard deviation 10). Linear regression analyses, corrected for age, sex, education, ethnicity and BMI, were used to study cross-sectional associations between OA and HRQoL. Data are presented as regression coefficients with $95 \%$ confidence intervals $(\mathrm{Cl})$. Because a suitable reference group without OA was lacking in the HOSTAS study, these patients were compared to the normative value of 50 . Previous research concluded a minimal clinically important difference of 2 points on the SF-36 scale, which was used to assess clinical relevance of differences in scores.

Results: Of the 6,334 NEO participants $8 \%$ were classified with only hand OA and $4 \%$ were classified concurrent hand and knee OA. The HOSTAS cohort consisted of a total of 538 patients with hand OA, of whom $57 \%$ fulfilled the ACR criteria for only hand $\mathrm{OA}$ and $32 \%$ was defined with concurrent hand and knee $\mathrm{OA}$. In the population-based NEO study, mean PCS was reduced with $-2.4(-3.6 ;-1.3)$ in participants with only hand OA, compared to participants without hand or knee OA (table 1). The subscales bodily pain and physical functioning were affected the most with mean differences of $-3.4(-4.6 ;-2.2)$ and $-2.1(-3.0 ;-1.1)$. Mental HRQoL was not reduced in participants with only hand $O A$, compared to participants without OA. In the population-based cohort $14 \%$ of participants with hand OA reported to have visited a medical specialist for OA. Participants with hand OA that have been referred to a medical specialist showed a lower physical HRQoL with a 